Ivo Senjanović

Marko Tomić

Nikola Vladimir

Neven Hadžić

DOI: $10.21278 /$ TOF.40301

ISSN 1333-1124

eISSN 1849-1391

\title{
AN ANALYTICAL SOLUTION TO FREE RECTANGULAR PLATE NATURAL VIBRATIONS BY BEAM MODES - ORDINARY AND MISSING PLATE MODES
}

\begin{abstract}
Summary
Relatively simple analytical procedures for the estimation of natural frequencies of free thin rectangular plates, based on the Rayleigh quotient and the Rayleigh-Ritz method, are presented. First, natural modes are assumed in the usual form as products of beam natural modes in the longitudinal and transverse directions, satisfying the grillage boundary conditions. Based on a detailed FEM analysis, the missing of some natural modes, defined as a sum and a difference of the cross products of beam modes, is noted. The frequencies of these modes are very similar and identical in some special cases, manifesting in such a way a double frequency phenomenon. These families of natural mode shapes form a complete natural frequency spectrum of a free rectangular plate as a novelty. The reliable approximation of natural modes enables the application of the Rayleigh quotient for the estimation of higher natural frequencies. The application of the developed procedure is illustrated by the case of a free thin square plate. The obtained results are compared with those determined by FEM and also with rigorous ones from the relevant literature based on the Rayleigh-Ritz method. The achieved accuracy is acceptable from the engineering point of view. Furthermore, the same problem is solved by the Rayleigh-Ritz method using approximate natural modes as mathematical ones. Direct and iterative procedures are presented. A small number of mathematical modes and iteration steps are sufficient to achieve reliable results.
\end{abstract}

Keywords: $\quad$ rectangular plate, natural vibration, Rayleigh quotient, Rayleigh-Ritz method, mode shapes, frequency spectrum

\section{Introduction}

A rectangular plate is a common structural element used in many engineering structures. The dynamic behaviour of thin and thick plates is analysed by the Kirchhoff [1] and the Mindlin theory [2]. For instance, the deck plating of a ship and the bulkhead grillages between cross girders are considered as thin plates, [3]. Also, Very Large Floating Structures (VLFS) like floating airports, artificial islands, etc. are analysed as large thin plates, [4]. Thick plates are usually used in mechanical engineering as engine supports. 
Vibration of a thin rectangular plate is a classical problem analysed in a large number of papers and has already been solved [5], while the vibrations of thick plates are still being investigated [6], [7]. In both cases, the analytical solution is achieved for a rectangular plate which is simply supported at least at two opposite edges. For some of the other combinations of simply supported and clamped edges, a rather complex, closed form solution is achieved, [8]. In addition, for all combinations of boundary conditions (simply supported, clamped, and free), a very complex approximate solution is presented in [9]. Because of that, numerical methods are preferable, as for instance the Rayleigh-Ritz method, or, more often, FEM due to its simplicity.

An analytical solution to free vibrations of a rectangular plate simply supported at two opposite edges is presented by Leissa, [10]. The problem of mixed boundary conditions (simply supported (S), clamped $(\mathrm{C})$, and free $(\mathrm{F})$ ) is analysed by the Rayleigh-Ritz method assuming the plate deflection as products of beam natural modes. The clamped and simply supported plate boundary conditions are completely satisfied, while the free edge conditions are only approximated, which reduces the accuracy of results. It is concluded that the additional symmetry of the square plate increases confusion when identifying natural modes. Certain vibration modes have not been discovered in the relevant literature.

The natural response of rectangular plates with free edges is analysed by Mizusawa, [11], by means of the Rayleigh-Ritz method with B-spline functions. The effects of Poisson's ratio on the natural frequencies of free-edge square plates are investigated. Malik and Bert [12] applied the differential quadrature element method to vibration analyses of plates with free boundary conditions, while Wang et al. [13] utilized a similar approach to both static and dynamic considerations of the above problem. In order to overcome the difficulty of implementing the free boundary conditions in the discrete singular convolution (DSC), Wei and his collaborators, [14] and [15], developed the method of matched interface and boundary (MIB), capable to analyse the first few natural frequencies. Also, the DSC method is applied by Wang and $\mathrm{Xu}[16]$ and comparisons with the above solutions are provided. Furthermore, in the context of applicability of related mathematical models to the analysis of the dynamic response of very large floating structures (VLFS), Wang et al. [17] highlighted some problems in obtaining accurate modal stress-resultant distributions in freely vibrating plates analysed by conventional methods. Namely, they showed that if one adopts the classical thin plate theory and the Galerkin method with commonly used modal functions consisting of the products of free-free beam modes, the natural boundary conditions are not satisfied at the free edges. Moreover, they indicated the persistence of the mentioned problem in the adoption of the refined Mindlin plate theory and the use of the NASTRAN software [18] (utilizing the finite element method) or the Ritz method. Furthermore, Wang et al. [17] demonstrated that a modified version of the Ritz method, which involves the penalty function for the enforcement of the natural boundary conditions, does not solve the problem when the plate is relatively thin due to the so-called artificial stiffening of the plate. In order to overcome the above problem, Wang et al. [19] proposed a mesh-free least squares-based finite difference method (LSFD) for evaluating vibration solutions of completely free plates, adopting not the classical but the Mindlin plate theory.

In the Rayleigh-Ritz method, natural modes are presented by polynomials with a large number of terms, which results in a rather time-consuming procedure. It is more effective to assume physical natural modes as a series of mathematical modes. For this purpose, products of beam natural modes in the longitudinal and transverse directions, satisfying the grillage boundary conditions, are usually used. In this case, one mathematical mode is dominant in each natural frequency. However, the FEM vibration analysis of a free square plate shows that there are some missing modes of specific shapes, which cannot be approximated by the product of beam modes successfully since none of the mathematical modes is dominant in this case. 
Due to the above reason, an analytical investigation into natural vibrations of free thin square and rectangular plates is carried out in [20]. Two additional families of missing mode shapes are identified and described by the sum and the difference of beam natural modes, respectively. The Rayleigh quotient is used not only for the first but also for the higher modes, [21]. In such a way, a complete and denser spectrum of natural frequencies is obtained.

In the present paper, the theory presented in [20] is summarised and the natural vibration analysis of a free rectangular plate is performed by the Rayleigh-Ritz method using approximate natural modes as mathematical ones. A direct numerical procedure and an iterative procedure are presented. The application is illustrated by the case of a square plate.

\section{Natural vibrations of free beam}

Natural modes of a free beam can be used in the vibration analysis of a free plate for the construction of mathematical modes. Due to practical reasons, it is suitable to distinguish between symmetric and antisymmetric modes. By satisfying the boundary conditions at beam ends $x= \pm a$, one obtains the following frequency equation for the symmetric modes, [20]

$$
\begin{aligned}
& \text { th } \alpha a+\operatorname{tg} \alpha a=0, \\
& \alpha_{0} a=0, \alpha_{2} a=2.3650, \alpha_{4} a=5.4978, \\
& \lim _{m \rightarrow \infty} \alpha_{m} a=\frac{1}{4}(2 m-1) \pi, m=0,2,4 \ldots,
\end{aligned}
$$

and the antisymmetric modes

$$
\begin{aligned}
& \text { th } \alpha a-\operatorname{tg} \alpha a=0, \\
& \alpha_{1} a=0, \alpha_{3} a=3.9266, \alpha_{5} a=7.0686, \\
& \lim _{m \rightarrow \infty} \alpha_{m} a=\frac{1}{4}(2 m-1) \pi, m=1,3,5 \ldots .
\end{aligned}
$$

The normalized symmetric and antisymmetric modes read

$$
\begin{aligned}
& X_{0}=1 \\
& X_{m}=\frac{1}{2}\left(\frac{\operatorname{ch} \alpha_{m} x}{\operatorname{ch} \alpha_{m} a}+\frac{\cos \alpha_{m} x}{\cos \alpha_{m} a}\right), m=2,4, \ldots, \\
& X_{1}=\frac{x}{a} \\
& X_{m}=\frac{1}{2}\left(\frac{\operatorname{sh} \alpha_{m} x}{\operatorname{sh} \alpha_{m} a}+\frac{\sin \alpha_{m} x}{\sin \alpha_{m} a}\right), m=3,5, \ldots
\end{aligned}
$$

Functions $X_{0}$ and $X_{1}$ represent rigid body modes, i.e. the beam translation and rotation. Index $m$ is the number of vibration nodes in a vibration mode. Mode functions $Y_{n}$ for the $y$ direction with the argument $\beta_{n} y$, where $-b \leq y \leq b$, can be written in an analogous way.

\section{Determination of free plate natural frequencies by the Rayleigh quotient}

\subsection{General}

The lowest natural frequency of a vibrating system can be determined in a relatively simple way by the Rayleigh quotient, (RQ) [5]. This approximate method involves the determination of the kinetic and potential (strain) energies of the considered system by using 
the assumed mode shape function which has to satisfy the geometric boundary conditions. Accuracy of the method depends on the success of approximation of the real physical natural mode by the assumed mathematical one. If it is possible to approximate successfully higher natural modes as the first one, the application of the Rayleigh quotient can be extended to the estimation of higher natural frequencies.

Strain energy of a vibrating plate, Fig. 1, is derived by integrating the work of bending and torsional moments on the corresponding curvature over the plate area, [5]. The resulting expression can be written in a suitable form for further use

$$
U=\frac{D}{2} \int_{-b}^{b} \int_{-a}^{a}\left[\left(\frac{\partial^{2} W}{\partial x^{2}}\right)^{2}+\left(\frac{\partial^{2} W}{\partial y^{2}}\right)^{2}+2 v \frac{\partial^{2} W}{\partial x^{2}} \frac{\partial^{2} W}{\partial y^{2}}+2(1-v)\left(\frac{\partial^{2} W}{\partial x \partial y}\right)^{2}\right] \mathrm{d} x \mathrm{~d} y
$$

where $W=W(x, y)$ is the natural mode, $D=E h^{3} /\left[12\left(1-v^{2}\right)\right]$ is the flexural rigidity, $E$ and $v$ are Young's modulus and Poisson's ratio, respectively. The first two terms are related to the bending in the $x$ and the $y$ direction, respectively, the third term takes the bending contraction into account, while the fourth term is a consequence of torsion.

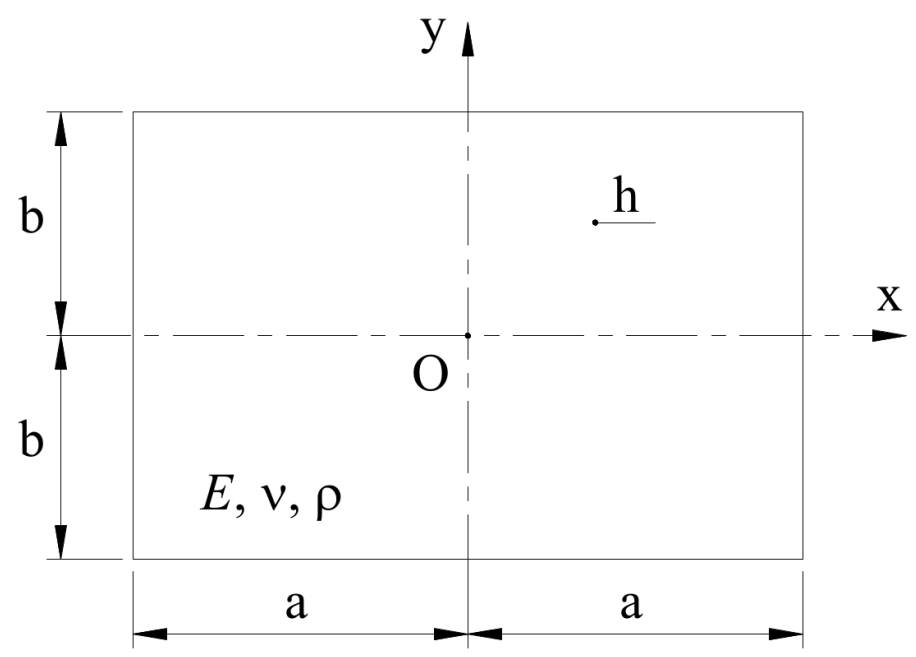

Fig. 1 Particulars of a thin rectangular plate

The kinetic energy takes quite a simple form

$$
K=\frac{1}{2} \omega^{2} \int_{-b}^{b} \int_{-a}^{a} m W^{2} \mathrm{~d} x \mathrm{~d} y
$$

where $m=\rho h$ is the mass per unit plate area and $\omega$ is the natural frequency.

It is well-known that the assumed mode shape function in the form of separated variables $W(x, y)=X(x) Y(y)$ captures all possible natural modes of a rectangular plate with any combination of simply supported and clamped edges. However, that is not the case if vibrations of a free plate are analysed. In order to investigate this fact, the FEM vibration analysis of a free square plate is performed and the first 24 natural modes are shown in Fig. 2. If the plate mode is assumed in the form $W_{m n}(x)=X_{m}(x) Y_{n}(y)$, where $m$ and $n$ are the numbers of beam vibration nodes, then the plate mode shape function has to manifest $m+n$ cross straight lines of zero displacement, Fig 3. Such a mesh can be noticed only in the modes 1,8 , and 17, and roughly in the modes $4,5,6,7,11,12,15,16,20,21$, and 24 in Fig. 2. As for all other remaining modes, it is necessary to find out another type of the plate mode shape functions. 
An Analytical Solution to

Free Rectangular Plate Natural Vibrations

by Beam Modes - Ordinary and Missing Plate Modes
I. Senjanović, M. Tomić, N. Vladimir, N. Hadžić

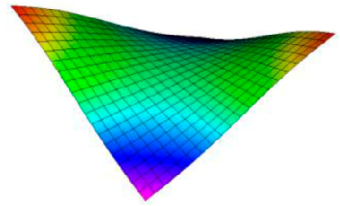

1

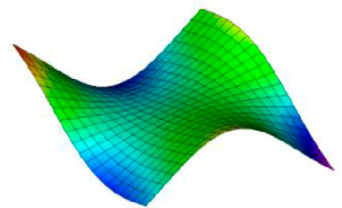

5

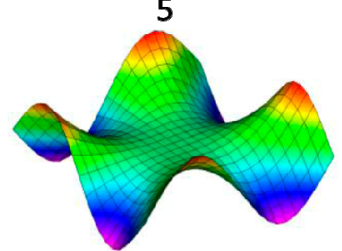

9

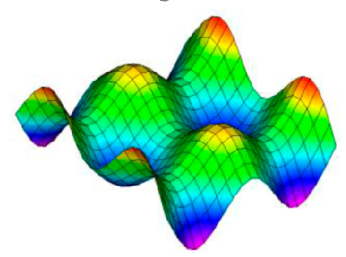

13

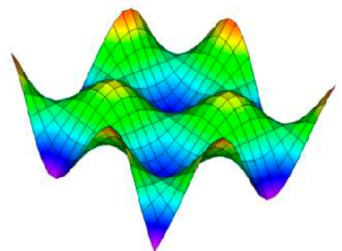

17

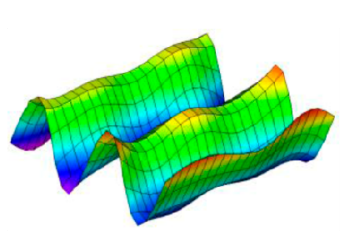

21

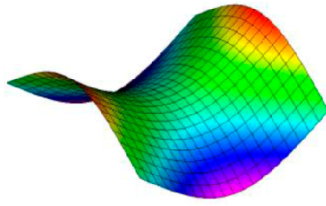

2

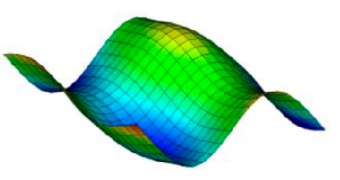

6

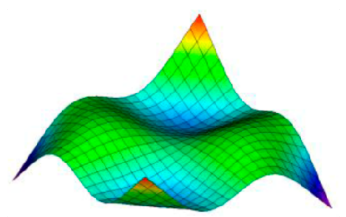

10

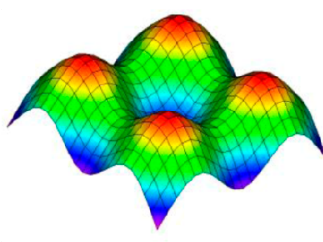

14

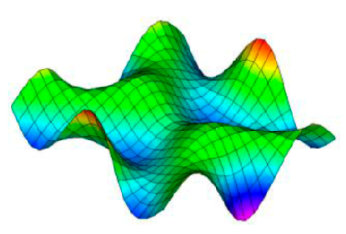

18

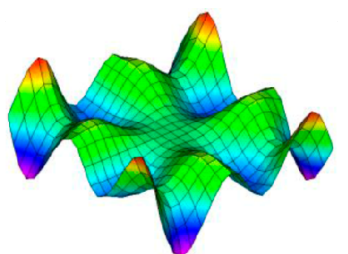

22

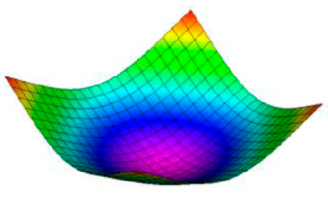

3

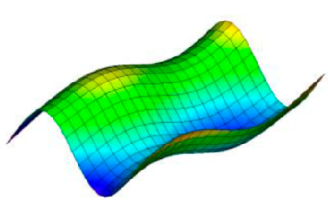

7

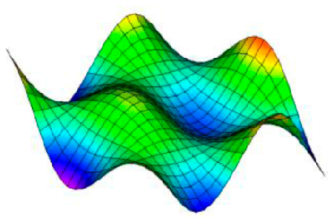

11

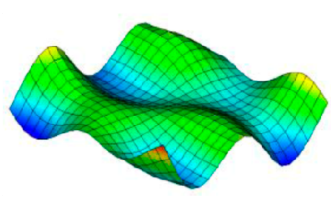

15

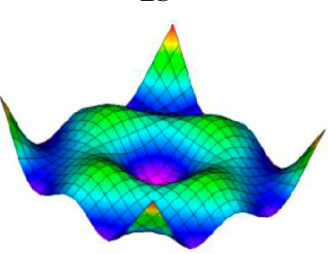

19

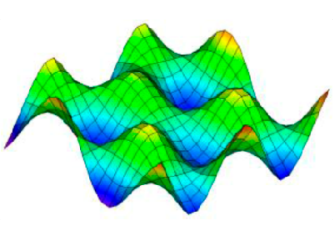

23
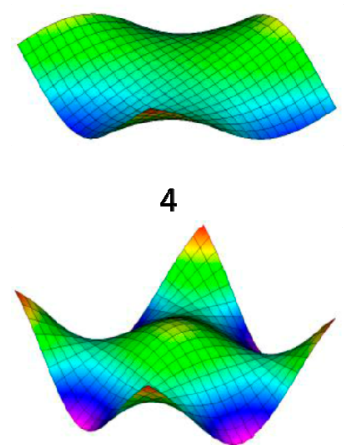

8

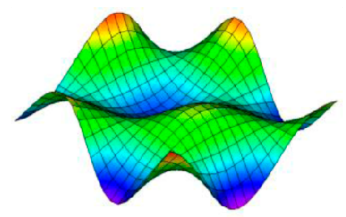

12

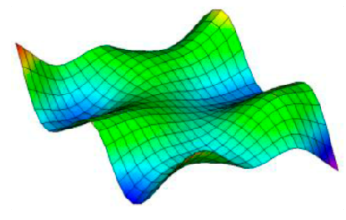

16

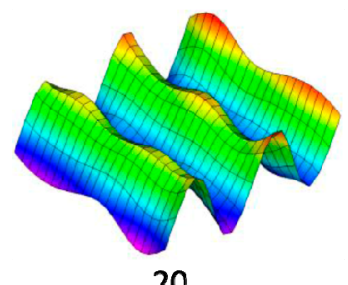

20

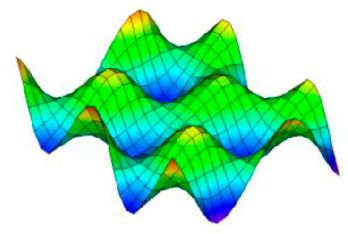

24

Fig. 2 Natural modes of a free square plate, FEM

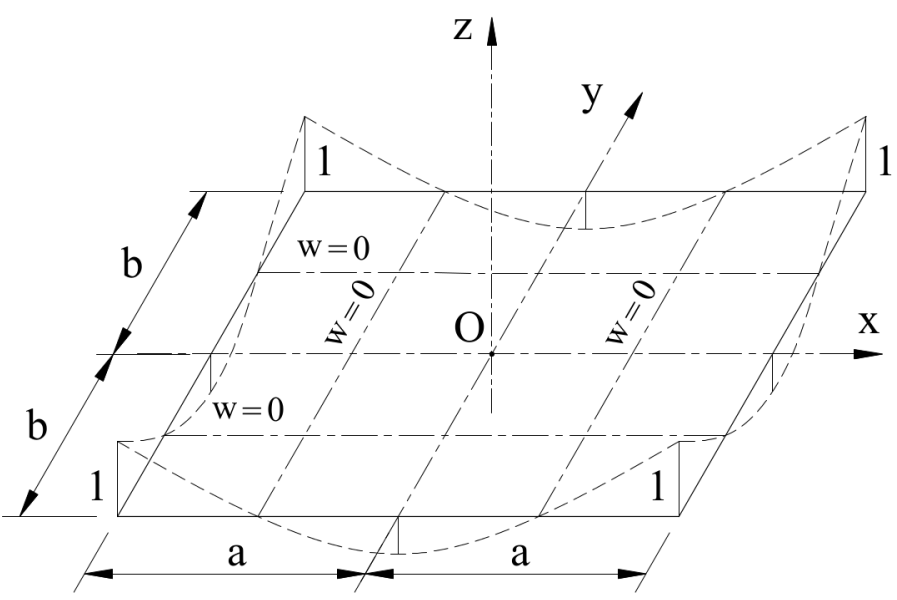

Fig. 3 An ordinary natural vibration mode of a free rectangular plate 


\subsection{Ordinary natural modes of a free plate}

In the usual procedure of the plate vibration analysis, the method of separation of variables is used, i.e. $W_{m n}(x, y)=X_{m}(x) Y_{n}(y)$. Eq. (5) for the strain energy takes the form

$$
U_{m n}=\frac{D}{2} \int_{-b}^{b} \int_{-a}^{a}\left[\left(X_{m}^{\prime \prime}\right)^{2} Y_{n}^{2}+X_{m}^{2}\left(Y_{n}^{\prime \prime}\right)^{2}+2 v X_{m}^{\prime \prime} X_{m} Y_{n}^{\prime \prime} Y_{n}+2(1-v)\left(X_{m}^{\prime} Y_{n}^{\prime}\right)^{2}\right] \mathrm{d} x \mathrm{~d} y
$$

For the coordinate function $X_{m}$, and analogously for $Y_{n}$, the beam mode functions (3) and (4), which satisfy the grillage boundary conditions, are usually used. In this case, all the integrals in (7) can be solved analytically as shown in [20]. By substituting the corresponding expressions for symmetric modes, Eq. (3) into (7), one obtains

$$
U_{m n}=\frac{D}{8 a b} u_{m n},
$$

where

$$
\begin{array}{r}
u_{m n}=\left(\alpha_{m}^{4}+\beta_{n}^{4}\right) a^{2} b^{2}+2 v \alpha_{m} a \beta_{n} b \text { th } \alpha_{m} a \text { th } \beta_{n} b\left(1-\alpha_{m} a \text { th } \alpha_{m} a\right)\left(1-\beta_{n} b \text { th } \beta_{n} b\right) \\
+2(1-v) \alpha_{m} a \beta_{n} b \text { th } \alpha_{m} a \text { th } \beta_{n} b\left(3+\alpha_{m} a \text { th } \alpha_{m} a\right)\left(3+\beta_{n} b \text { th } \beta_{n} b\right) .
\end{array}
$$

In the case of uniform mass distribution, the expression for the plate kinetic energy (6) takes a very simple form

$$
K_{m n}=\frac{1}{2} \omega^{2} m \int_{-b}^{b} \int_{-a}^{a} X_{m}^{2} Y_{n}^{2} \mathrm{~d} x \mathrm{~d} y=\frac{1}{8} \omega^{2} m a b
$$

Due to the energy balance, $U=K$, the natural frequency can be determined by the Rayleigh quotient

$$
\omega_{m n}^{2}=\frac{D}{m} \frac{u_{m n}}{a^{2} b^{2}}
$$

In the case of antisymmetric beam modes $X_{m}$ or/and $Y_{n}$, the same structure of expressions (8) and (9) for the strain energy are valid, but the corresponding hyperbolic function th(.) has to be replaced with $\operatorname{cth}($.$) . Hence, the kinetic energy (10) is valid for all$ single and double symmetric and antisymmetric plate modes.

\subsection{Missing natural modes of a free plate}

Observing the shape of modes 2 and 3 in Fig. 2, one can notice that the former is similar to a hyperbolic paraboloid (saddle), while the latter is similar to an elliptical paraboloid. These paraboloids are mathematically described in a normalized form as

$$
z_{h}=\frac{x^{2}}{a^{2}}-\frac{y^{2}}{b^{2}}, \quad z_{e}=\frac{x^{2}}{a^{2}}+\frac{y^{2}}{b^{2}}
$$

where parameters $a$ and $b$ are the plate dimensions as shown in Fig. 1. In both cases, crosssections of paraboloids, parallel to the planes $x 0 z$ and $y 0 z$, are parabolas.

Based on the noted similarity of modes 2 and 3 to paraboloids, Fig. 2, one can write

$$
W_{2 \pm 2}=X_{2} \pm Y_{2}
$$


since the shapes of the beam functions $X_{2}$ and $Y_{2}$ look like parabolas $x^{2}$ and $y^{2}$ in (12). Moreover, numerical examples show that the missing modes can be assumed in a more complex general form

$$
W_{m n \pm k l}=X_{m} Y_{n}+s X_{k} Y_{l}
$$

where $s= \pm 1$ is the sign for the definition of the elliptical and the hyperbolic mode shape.

By substituting (14) into (5), one obtains the following expression for the strain energy

$$
\begin{aligned}
U_{m n \pm k l}= & \frac{D}{2} \int_{-b}^{b} \int_{-a}^{a}\left[\left(X_{m}^{\prime \prime} Y_{n}+s X_{k}^{\prime \prime} Y_{l}\right)^{2}+\left(X_{m} Y_{n}^{\prime \prime}+s X_{k} Y_{l}^{\prime \prime}\right)^{2}\right. \\
& \left.+2 v\left(X_{m}^{\prime \prime} Y_{n}+s X_{k}^{\prime \prime} Y_{l}\right)\left(X_{m} Y_{n}^{\prime \prime}+s X_{k} Y_{l}^{\prime \prime}\right)+2(1-v)\left(X_{m}^{\prime} Y_{n}^{\prime}+s X_{k}^{\prime} Y_{l}^{\prime}\right)^{2}\right] \mathrm{d} x \mathrm{~d} y .
\end{aligned}
$$

Expansion of Eq. (15) leads to the form of separated integrals

$$
\begin{aligned}
U_{m n \pm k l}= & \frac{D}{2} \int_{-b}^{b} \int_{-a}^{a}\left\{\left(X_{m}^{\prime \prime}\right)^{2} Y_{n}^{2}+\left(X_{k}^{\prime \prime}\right)^{2} Y_{l}^{2}+X_{m}^{2}\left(Y_{n}^{\prime \prime}\right)^{2}+X_{k}^{2}\left(Y_{l}^{\prime \prime}\right)^{2}\right. \\
& +2 v\left[X_{m}^{\prime \prime} X_{m} Y_{n}^{\prime \prime} Y_{n}+s\left(X_{m} X_{k}^{\prime \prime} Y_{n}^{\prime \prime} Y_{l}+X_{m}^{\prime \prime} X_{k} Y_{n} Y_{l}^{\prime \prime}\right)+X_{k}^{\prime \prime} X_{k} Y_{l}^{\prime \prime} Y_{l}\right] \\
& \left.+2(1-v)\left[\left(X_{m}^{\prime}\right)^{2}\left(Y_{n}^{\prime}\right)^{2}+2 s X_{m}^{\prime} X_{k}^{\prime} Y_{n}^{\prime} Y_{l}^{\prime}+\left(X_{k}^{\prime}\right)^{2}\left(Y_{l}^{\prime}\right)^{2}\right]\right\} \mathrm{d} x \mathrm{~d} y .
\end{aligned}
$$

where $s^{2}=1$ is taken into account. Also, two zero terms in (16), due to the orthogonality of the beam modal functions, are omitted.

On the other hand, by substituting (14) into (6), the kinetic energy reads

$$
K_{m n \pm k l}=\frac{1}{2} \omega_{m n \pm k l}^{2} m \int_{-b}^{b} \int_{-a}^{a}\left(X_{m}^{2} Y_{n}^{2}+X_{k}^{2} Y_{l}^{2}\right) \mathrm{d} x \mathrm{~d} y
$$

where one term is excluded due to the orthogonality of the beam modal functions.

All the integrals in Eq. (16) and (17) can be solved analytically by employing the beam modal functions $X_{m}$ and $Y_{n}$, Eq. (3) and (4). Solutions of typical integrals are given in [20]. Hence, one finds the expression for the kinetic energy

$$
K_{m n \pm k l}=\frac{1}{4} \omega_{m n \pm k l}^{2} m a b
$$

Finally, the balance of the strain and kinetic energy leads to

$$
\omega_{m n \pm k l}^{2}=\frac{4 U_{m n \pm k l}}{m a b}
$$

\subsection{Natural vibrations of a square plate}

Reliability of the developed theory and the accuracy of numerical results are checked in the case of natural vibrations of a free thin square plate, where $a=b$. Dimensionless frequency parameter is specified as

$$
\Omega=\omega \frac{(2 b)^{2}}{\pi^{2}} \sqrt{\frac{m}{D}}
$$


The frequency parameter (20) for ordinary plate natural modes, $W_{m n}=X_{m} Y_{n}$, by employing (11), is presented in the form

$$
\Omega\left(X_{m} Y_{n}\right)=\frac{4}{\pi^{2}}\left(\frac{b}{a}\right) \sqrt{\mathrm{u}_{m n}}
$$

where $u_{m n}$ is defined by (9).

In a similar way, the frequency parameter for the missing plate natural modes, Eq. (14), by taking into account Eqs. (16) and (19), can be presented in the form

$$
\Omega\left(X_{m} Y_{n} \pm X_{k} Y_{l}\right)=\frac{4 \sqrt{2}}{\pi^{2}}\left(\frac{b}{a}\right) \sqrt{a b I_{m n \pm k l}}
$$

where $I_{m n \pm k l}$ is the surface integral in expression (16).

It is necessary to point out that expressions (21) and (22) are valid only for elastic beam modes. If a rigid body mode is involved, expressions for the frequency parameter have to be derived from the very beginning of energy formulation.

Natural vibrations of a square plate, Fig. 1, are calculated by FEM, using the NASTRAN software [18]. The first 24 natural modes are shown in Fig. 2, and the corresponding values of frequency parameter are listed in Table 1. Also, results from the relevant literature, determined by the more rigorous Rayleigh-Ritz method for the first 10 modes, [22], are given for correlation.

Values of the frequency parameter determined by the present procedure (PP) are included in Table 1, with the indication of the mode type. Additional explanation for each of the first 14 natural modes is given in [20].

Analytically defined approximate natural modes are shown in Fig. 4. They are of the same global shape as those obtained numerically by FEM. The FEM natural frequencies are slightly underestimated, while those of the present procedure are overestimated, Table 1. Discrepancies of FEM results with respect to the rigorous solutions for the first 10 modes, [22], are within $0.5 \%$, and those of the present procedure are within $6 \%$.

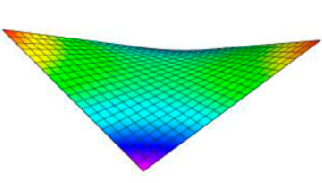

1

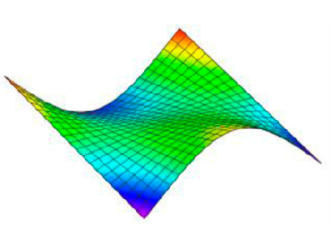

5

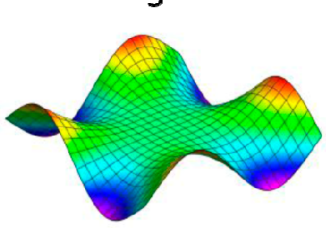

9

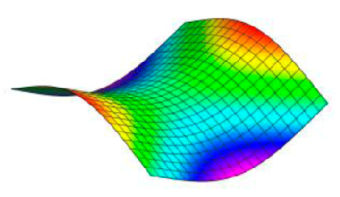

2
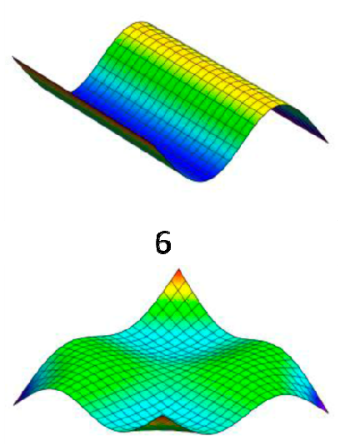

10

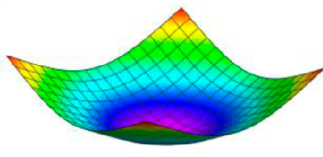

3

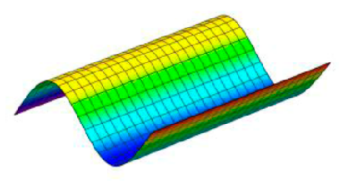

7

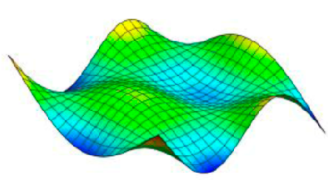

11
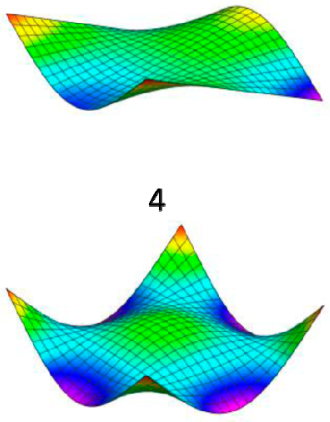

8

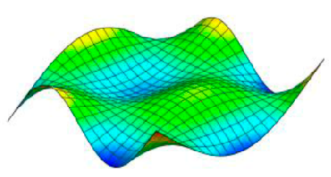

12 
An Analytical Solution to

Free Rectangular Plate Natural Vibrations

by Beam Modes - Ordinary and Missing Plate Modes
I. Senjanović, M. Tomić,

N. Vladimir, N. Hadžić

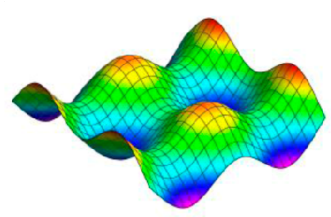

13
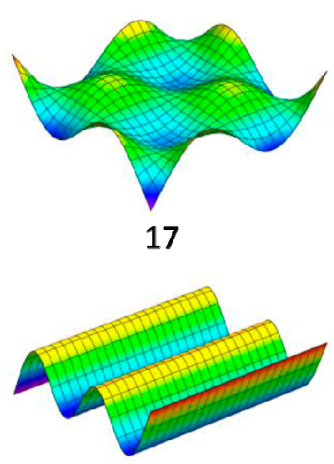

21
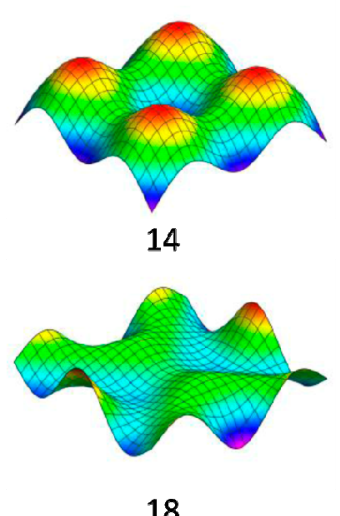

18

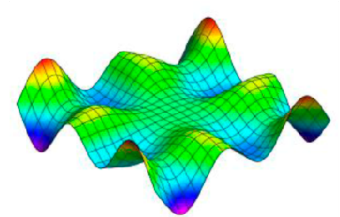

22

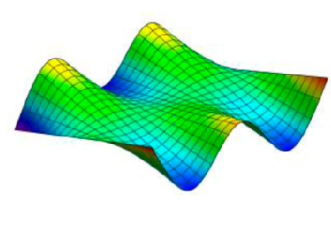

15

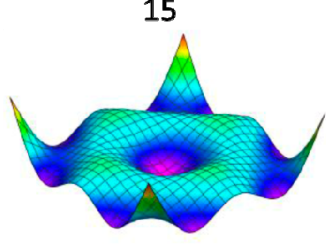

19

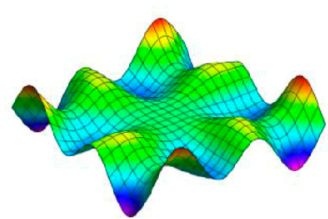

23
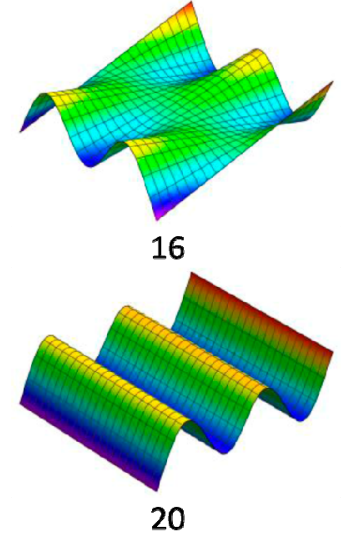

20

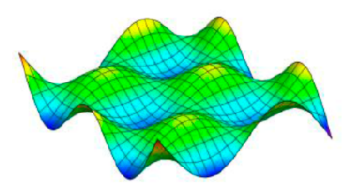

24

Fig. 4 Analytical natural modes of a square plate

Table 1 Frequency parameter $\Omega=\omega(2 b / \pi)^{2} \sqrt{m / D}$ of a free square plate, approximate analytical solution

\begin{tabular}{ccccccc}
\hline Mode no. & Mode type & FEM & Discrepancy, $\%$ & RQ, PP & Discrepancy, \% & RRM, Liew [22] \\
\hline 1 & $X_{1} Y_{1}$ & 1.3631 & -0.11 & 1.4386 & 5.42 & 1.3646 \\
2 & $X_{2} Y_{0}-X_{0} Y_{2}$ & 1.9827 & -0.15 & 2.0186 & 1.66 & 1.9856 \\
3 & $X_{2} Y_{0}+X_{0} Y_{2}$ & 2.4564 & -0.11 & 2.4906 & 1.28 & 2.4590 \\
4 & $X_{1} Y_{2}$ & 3.5185 & -0.21 & 3.6977 & 4.87 & 3.5260 \\
5 & $X_{2} Y_{1}$ & 3.5185 & -0.21 & 3.6977 & 4.87 & 3.5260 \\
6 & $X_{3} Y_{0}-X_{0} Y_{3}$ & 6.1772 & -0.21 & 6.2488 & 0.95 & 6.1900 \\
7 & $X_{3} Y_{0}+X_{0} Y_{3}$ & 6.1772 & -0.21 & 6.2488 & 0.95 & 6.1900 \\
8 & $X_{2} Y_{2}$ & 6.4317 & -0.32 & 6.8116 & 5.56 & 6.4526 \\
9 & $X_{3} Y_{1}-X_{1} Y_{3}$ & 6.9930 & -0.35 & 7.0710 & 0.76 & 7.0179 \\
10 & $X_{3} Y_{1}+X_{1} Y_{3}$ & 7.7798 & -0.50 & 8.1036 & 3.64 & 7.8190 \\
11 & $X_{2} Y_{3}$ & 10.6340 & & 11.1865 & $(5.20)^{*}$ & \\
12 & $X_{3} Y_{2}$ & 10.6340 & & 11.1865 & $(5.20)^{*}$ & \\
13 & $X_{4} Y_{0}-X_{0} Y_{4}$ & 11.8280 & & 12.0045 & $(1.49)^{*}$ & \\
14 & $X_{4} Y_{0}+X_{0} Y_{4}$ & 12.3720 & & 12.4909 & $(0.96)^{*}$ & \\
15 & $X_{1} Y_{4}$ & 13.2683 & & 13.5016 & $(1.76)^{*}$ & \\
16 & $X_{4} Y_{1}$ & 13.2683 & & 13.5016 & $(1.76)^{*}$ & \\
17 & $X_{3} Y_{3}$ & 15.3853 & & 16.1765 & $(5.14)^{*}$ & \\
18 & $X_{4} Y_{2}-X_{2} Y_{4}$ & 16.2566 & & 16.4035 & $(0.90)^{*}$ & \\
19 & $X_{4} Y_{2}+X_{2} Y_{4}$ & 16.9757 & & 17.9472 & $(5.72)^{*}$ & \\
20 & $X_{0} Y_{5}$ & 20.0104 & & 20.2500 & $(1.20)^{*}$ & \\
21 & $X_{5} Y_{0}$ & 20.0104 & & 20.2500 & $(1.20)^{*}$ & \\
22 & $X_{5} Y_{1}-X_{1} Y_{5}$ & 20.5749 & & 20.8477 & $(1.33)^{*}$ & \\
23 & $X_{5} Y_{1}+X_{1} Y_{5}$ & 21.5824 & & 21.9931 & $(1.90)^{*}$ & \\
24 & $X_{4} Y_{3}$ & 21.6451 & & 22.5767 & $(4.30)^{*}$ & \\
25 & $X_{3} Y_{4}$ & 21.6451 & & 22.5767 & $(4.30)^{*}$ & \\
\hline
\end{tabular}

* With respect to FEM 


\section{Free plate natural vibration analysis by the Rayleigh-Ritz method}

\subsection{Direct procedure}

In the Rayleigh-Ritz method, a natural mode is assumed in the form of a series

$$
W(x, y)=\sum_{j=1}^{\infty} A_{j} Z_{j}(x, y),
$$

where $A_{j}$ are the unknown constants and $Z_{j}(x, y)$ are the appropriate deflection functions, which individually have to satisfy at least the geometric boundary conditions. Functions $Z_{j}(x, y)$ are usually called mathematical modes, while $W(x, y)$ is a physical mode.

The modal strain energy of a vibrating plate has to be equal to the kinetic energy. For an approximate natural mode, the difference has to be minimal. By substituting (23) into (5) and (6) and satisfying the condition

$$
\frac{\partial U}{\partial A_{i}}-\frac{\partial K}{\partial A_{i}}=0
$$

one arrives at the system of homogenous algebraic equations

$$
\left(\left[S_{i j}^{0}\right]-\omega^{2}\left[M_{i j}^{0}\right]\right)\left\{A_{j}\right\}=\{0\}
$$

where

$$
\begin{aligned}
& S_{i j}^{0}=D \int_{-b}^{b} \int_{-a}^{a}[ \frac{\partial^{2} Z_{i}}{\partial x^{2}} \frac{\partial^{2} Z_{j}}{\partial x^{2}}+\frac{\partial^{2} Z_{i}}{\partial y^{2}} \frac{\partial^{2} Z_{j}}{\partial y^{2}}+v\left(\frac{\partial^{2} Z_{i}}{\partial x^{2}} \frac{\partial^{2} Z_{j}}{\partial y^{2}}+\frac{\partial^{2} Z_{i}}{\partial y^{2}} \frac{\partial^{2} Z_{j}}{\partial x^{2}}\right) \\
&\left.+2(1-v) \frac{\partial^{2} Z_{i}}{\partial x \partial y} \frac{\partial^{2} Z_{j}}{\partial x \partial y}\right] \mathrm{d} x \mathrm{~d} y \\
& M_{i j}^{0}=m \int_{-b}^{b} \int_{-a}^{a} Z_{i} Z_{j} \mathrm{~d} x \mathrm{~d} y
\end{aligned}
$$

are the elements of the modal stiffness and mass matrix, respectively. Once the spectrum of natural frequencies $\omega_{p}, p=1,2 \ldots$, for a chosen number of terms in the series (23) is determined from the condition

$$
\operatorname{Det}\left(\left[S_{i j}^{0}\right]-\omega^{2}\left[M_{i j}^{0}\right]\right)=0,
$$

the corresponding vector of constants $A_{p j}$ for each $\omega_{p}$ is obtained from (25) by assuming the unit value for one of the constants. An appropriate choice is to take $A_{p p}=1$ since it is expected to be dominant.

The mathematical modes for a free plate in (23) are usually assumed in the form of a product of free beam natural modes, Eq. (3) and (4)

$$
Z_{i}(x, y)=\left[X_{m}(x) Y_{n}(y)\right]_{i}, \quad Z_{j}(x, y)=\left[X_{k}(x) Y_{l}(y)\right]_{j},
$$

where $m, n=1,2 \ldots M$ and $k, l=1,2 \ldots N$. The indices $i$ and $j$ are prescribed to each combination of indices $m$ and $n$, and $k$ and $l$, respectively. By substituting (29) into (26), one gets 


$$
\begin{aligned}
S_{i j}^{0}=D \int_{-b}^{b} \int_{-a}^{a} & {\left[\left(X_{m}^{\prime \prime} X_{k}^{\prime \prime} Y_{n} Y_{l} \delta_{n l}\right)_{i j}+\left(X_{m} X_{k} Y_{n}^{\prime \prime} Y_{l}^{\prime \prime} \delta_{m k}\right)_{i j}\right.} \\
& \left.+v\left(X_{m}^{\prime \prime} X_{k} Y_{n} Y_{l}^{\prime \prime}+X_{m} X_{k}^{\prime \prime} Y_{n}^{\prime \prime} Y_{l}\right)_{i j}+2(1-v)\left(X_{m}^{\prime} X_{k}^{\prime} Y_{n}^{\prime} Y_{l}^{\prime}\right)_{i j}\right] \mathrm{d} x \mathrm{~d} y .
\end{aligned}
$$

The beam modes are orthogonal and that is indicated in (30) by the Kronecker symbol. If $l=n$, then $k=m$ and the first two terms in (30) are diagonal

$$
f_{1}(x, y)_{i j}+f_{2}(x, y)_{i j}=\left[\left(X_{m}^{\prime \prime}\right)^{2} Y_{n}^{2}+X_{m}^{2}\left(Y_{n}^{\prime \prime}\right)^{2}\right] \delta_{i j}
$$

All integrals in (31) per $x$ and $y$ can be solved analytically. For the first two integrals, by employing the expressions from [20], one obtains

$$
\left(I_{1}+I_{2}\right)_{i j}=\frac{a b}{4}\left(\alpha_{m}^{4}+\beta_{n}^{4}\right) \delta_{i j}
$$

The third and fourth terms in (30) generate a full matrix.

By substituting the expressions (29) for the mathematical modes into (27), the mass matrix reads

$$
M_{i j}^{0}=m \int_{-b}^{b} \int_{-a}^{a}\left[X_{m} X_{k} Y_{n} Y_{l}\right]_{i j} \mathrm{~d} x \mathrm{~d} y
$$

Due to the orthogonality of the beam natural modes, the mass matrix is diagonal

$$
M_{i j}^{0}=m \int_{-b}^{b} \int_{-a}^{a} X_{m}^{2} Y_{n}^{2} \mathrm{~d} x \mathrm{~d} y \delta_{i j}
$$

After integration, according to the expressions given in [20], one gets

$$
M_{i j}^{0}=\frac{1}{4} m a b \delta_{i j}
$$

Hence, elements of the diagonal mass matrix are the same for all modes.

If the matrix equation of natural vibrations (25) for the $p$-th mode is multiplied by the vector $\left\{A_{p i}\right\}^{T}$ from the left side, taking (35) and $A_{p p}=1$ into account, one obtains

$$
\left\langle A_{p i}\right\rangle\left[S_{i j}^{0}\right]\left\{A_{p j}\right\}-\omega_{p}^{2} \frac{1}{4} \operatorname{mab}\left\langle A_{p j}\right\rangle\left\{A_{p j}\right\}=0
$$

The first and the second term in (36) are the condensed stiffness and the mass of the $p$-th mode, respectively, as a result of the modes coupling. Equation (36) can be written in the algebraic form

$$
\sum_{i=1}^{n} \sum_{j=1}^{n} A_{p i} S_{i j}^{0} A_{p j}-\omega_{p}^{2} \frac{1}{4} m a b \sum_{j=1}^{n} A_{p j}^{2}=0
$$

Expression (37) can be used for the benchmarking of results.

\subsection{Iterative procedure}

The eigenvalue formulation for determining a direct solution to the plate natural vibration, Eq. (25), is specific since the diagonal terms of stiffness matrix are predominant, while the mass matrix is diagonal. In that case, the procedure for the eigenvalue solution can be simplified by employing an iterative procedure. 
The matrix equation of natural vibration (25) with the full stiffness matrix and diagonal mass matrix can be presented in the expanded form

$$
\left[\begin{array}{cccccc}
S_{11}^{0}-\omega^{2} M & S_{12}^{0} & \cdots & S_{1 i}^{0} & \cdots & S_{1 n}^{0} \\
S_{21}^{0} & S_{22}^{0}-\omega^{2} M & \cdots & S_{2 i}^{0} & \cdots & S_{2 n}^{0} \\
\cdots & \cdots & \cdots & \cdots & \cdots & \cdots \\
S_{i 1}^{0} & S_{i 2}^{0} & \cdots & S_{i i}^{0}-\omega^{2} M & \cdots & S_{i n}^{0} \\
\cdots & \cdots & \cdots & \cdots & \cdots & \cdots \\
S_{n 1}^{0} & S_{n 2}^{0} & \cdots & S_{n i}^{0} & \cdots & S_{n n}^{0}-\omega^{2} M
\end{array}\right]\left\{\begin{array}{c}
A_{1} \\
A_{2} \\
\cdots \\
A_{i} \\
\cdots \\
A_{n}
\end{array}\right\}=\{0\}
$$

where, according to (35), $M=m a b / 4$.

The natural frequency of the $i$-th mode consists of two parts.

$$
\omega_{i}^{2}=\left(\omega_{i}^{0}\right)^{2}+\left(\omega_{i}^{*}\right)^{2}
$$

where $\omega_{i}^{0}$ is the approximate value of natural frequency and $\omega_{i}^{*}$ is the correction due to the coupling of mathematical modes. The approximate natural frequency is determined by setting the diagonal element of the $i$-th equation in (38) to zero, i.e. $\left(\omega_{i}^{0}\right)^{2}=S_{i i}^{0} / M$, Eq. (11). Furthermore, $\left(\omega_{i}^{0}\right)^{2}$ is inserted into (38), the unit value is chosen for the dominant constant, $A_{i i}=1$, the $i$-th row is excluded from (38) and the $i$-th column, with the known elements $S_{k i}^{0} A_{i i}, k=1,2, \ldots n$ is transferred from the left to the right hand side. As a result, the following reduced nonhomogeneous matrix equation is obtained

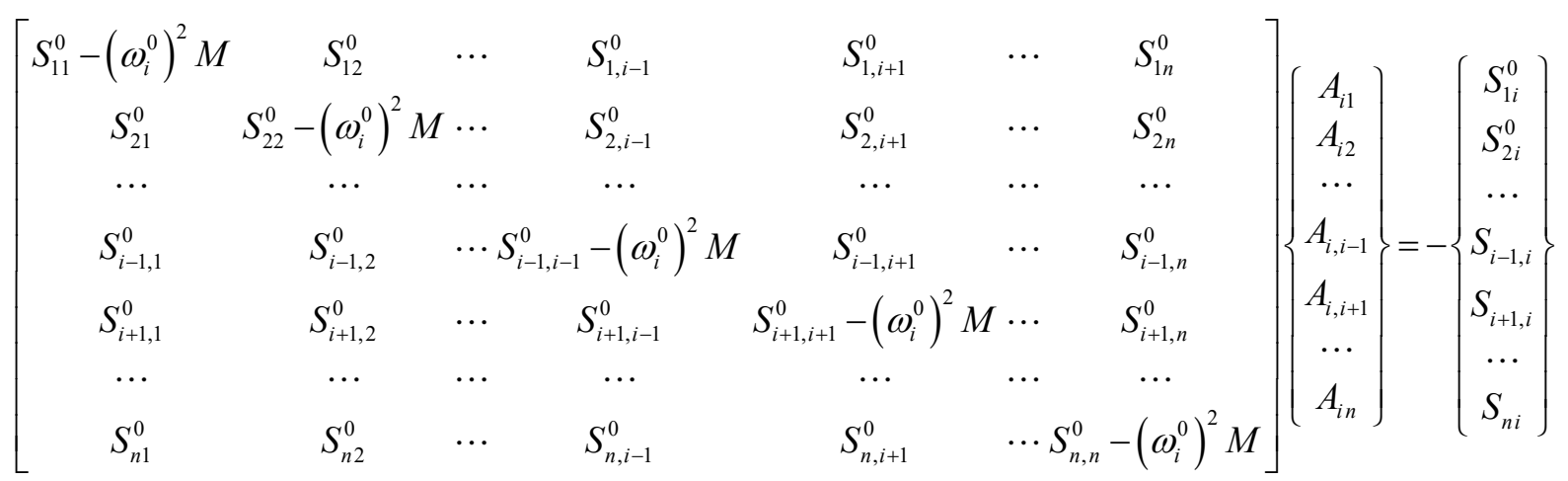

Solving (39) and adding $A_{i i}=1$, the vector of coefficients of the $i$-th mode is defined. By substituting (39) into the $i$-th equation in (38), the additional part of the natural frequency is obtained

$$
\left(\omega_{i}^{*}\right)^{2}=\frac{1}{M} \sum_{j=1}^{n} A_{i j} S_{i j}^{0}\left(1-\delta_{i j}\right)
$$

Now, $\left(\omega_{i}^{(1)}\right)^{2}=\left(\omega_{i}^{0}\right)^{2}+\left(\omega_{i}^{*(1)}\right)^{2}$ from the first step is inserted into (40) and new values of the constants vector $\left\{A_{i j}^{(1)}\right\}$ are determined from (40). The iteration is continued until the stabilization of $\omega_{i}^{(k)}$ is achieved. 


\subsection{Illustrative example}

Natural frequencies of a free square plate are determined by the Rayleigh-Ritz method in two ways:

1. Direct procedure, 25 ordinary and missing modes from Table 1, (DP-OMm),

2. Iterative procedure, 25 ordinary and missing modes from Table 1, the first step, (IP-OMm).

Table 2 presents a comparison between the results obtained for the first 10 modes and with those from [22], which are calculated by the Rayleigh-Ritz method, taking a large number of power series terms into account. The IP-OMm values, determined by only one iteration step, are very close to the DP-OMm values, and, for some modes, are slightly more accurate. Differences in the values of both frequency parameters with respect to the rigorous solution, Liew [22], are within 1\%. The diagram in Fig. 5 shows that the accuracy of IP-OMm frequency parameters with one iteration step is considerably increased with respect to the approximate analytical RQ solution. It is interesting that, observing the structure of the stiffness matrix, one can notice that each mode is coupled with only a few other modes.

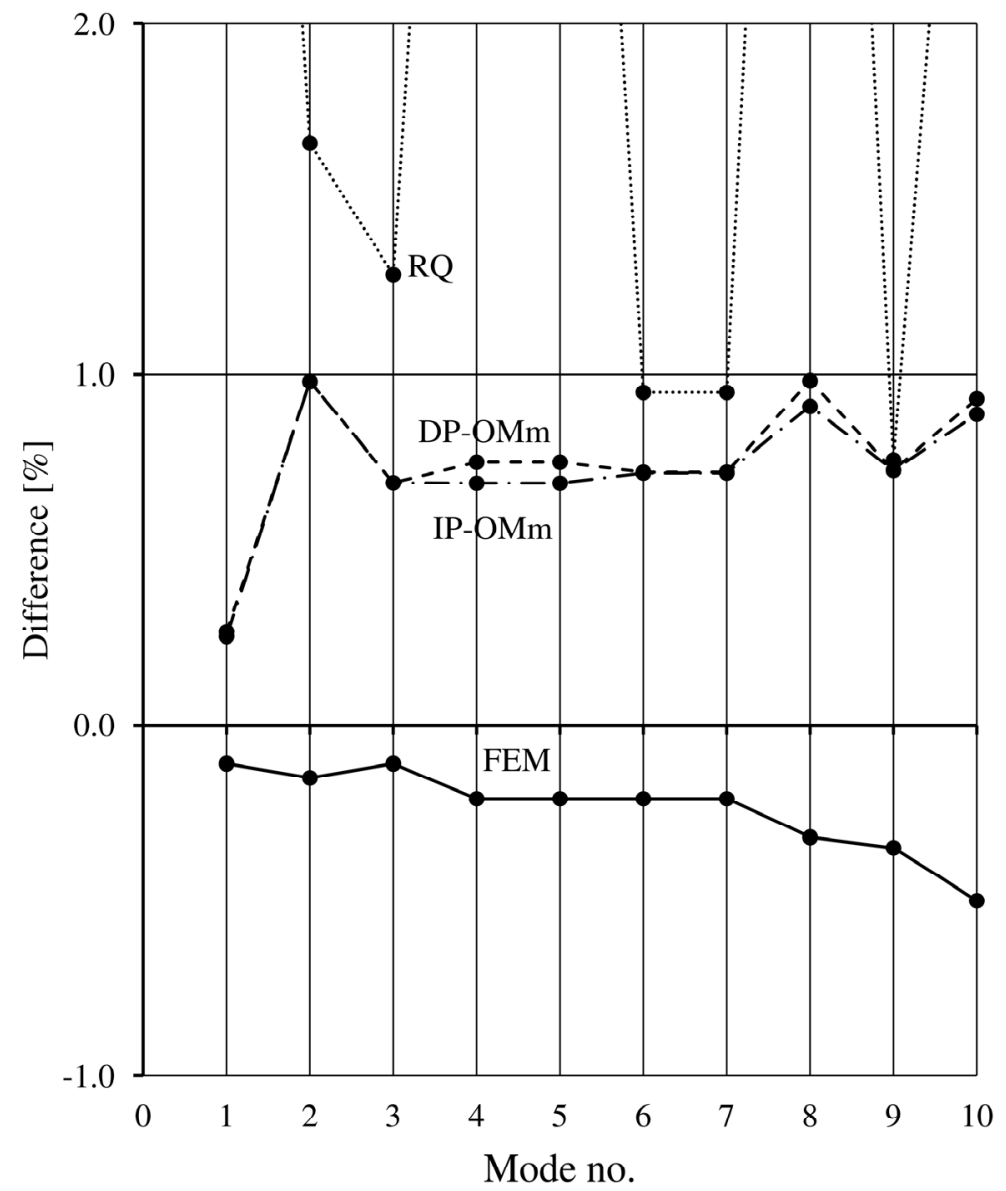

Fig. 5 Differences between various frequency parameters and the RRM values of a free square plate 
Table 2 Frequency parameter $\Omega=\omega(2 b / \pi)^{2} \sqrt{m / D}$ of a free square plate, rigorous solutions, 25 approximate modes from Table 1

\begin{tabular}{cccccc}
\hline Mode no. & DP-OMm & Difference, $\%$ & IP-OMm & Difference, $\%$ & RRM, Liew, [22] \\
\hline 1 & 1.3682 & 0.264 & 1.3680 & 0.249 & 1.3646 \\
2 & 2.0051 & 0.982 & 2.0051 & 0.982 & 1.9856 \\
3 & 2.4760 & 0.691 & 2.4760 & 0.691 & 2.4590 \\
4 & 3.5524 & 0.749 & 3.5503 & 0.689 & 3.5260 \\
5 & 3.5524 & 0.749 & 3.5503 & 0.689 & 3.5260 \\
6 & 6.2347 & 0.722 & 6.2345 & 0.719 & 6.1900 \\
7 & 6.2347 & 0.722 & 6.2345 & 0.719 & 6.1900 \\
8 & 6.5160 & 0.982 & 6.5112 & 0.908 & 6.4526 \\
9 & 7.0689 & 0.727 & 7.0689 & 0.727 & 7.0179 \\
10 & 7.8918 & 0.931 & 7.8883 & 0.886 & 7.8190 \\
\hline
\end{tabular}

In order to investigate the convergence of frequency parameters determined by the Rayleigh-Ritz method and analytical mathematical modes, DP-OMm, three sets of the mode series up to the maximal numbers of mode indices $\mathrm{M}=\mathrm{N}=4,5$, and 6 are taken into account. Ordinary modes which are constitutive parts of missing modes are excluded from the analysis in order to avoid their double contribution. The obtained results are listed in Table 3 and shown in Fig. 6, and the convergence of the procedure is obvious. The diagram of the DPOMm values in Fig. 5, determined by the 25 ordinary and missing modes, is close to the diagram of DP-OMm values in Fig. 6 , obtained by using up to $\mathrm{M}=\mathrm{N}=5$ modes.

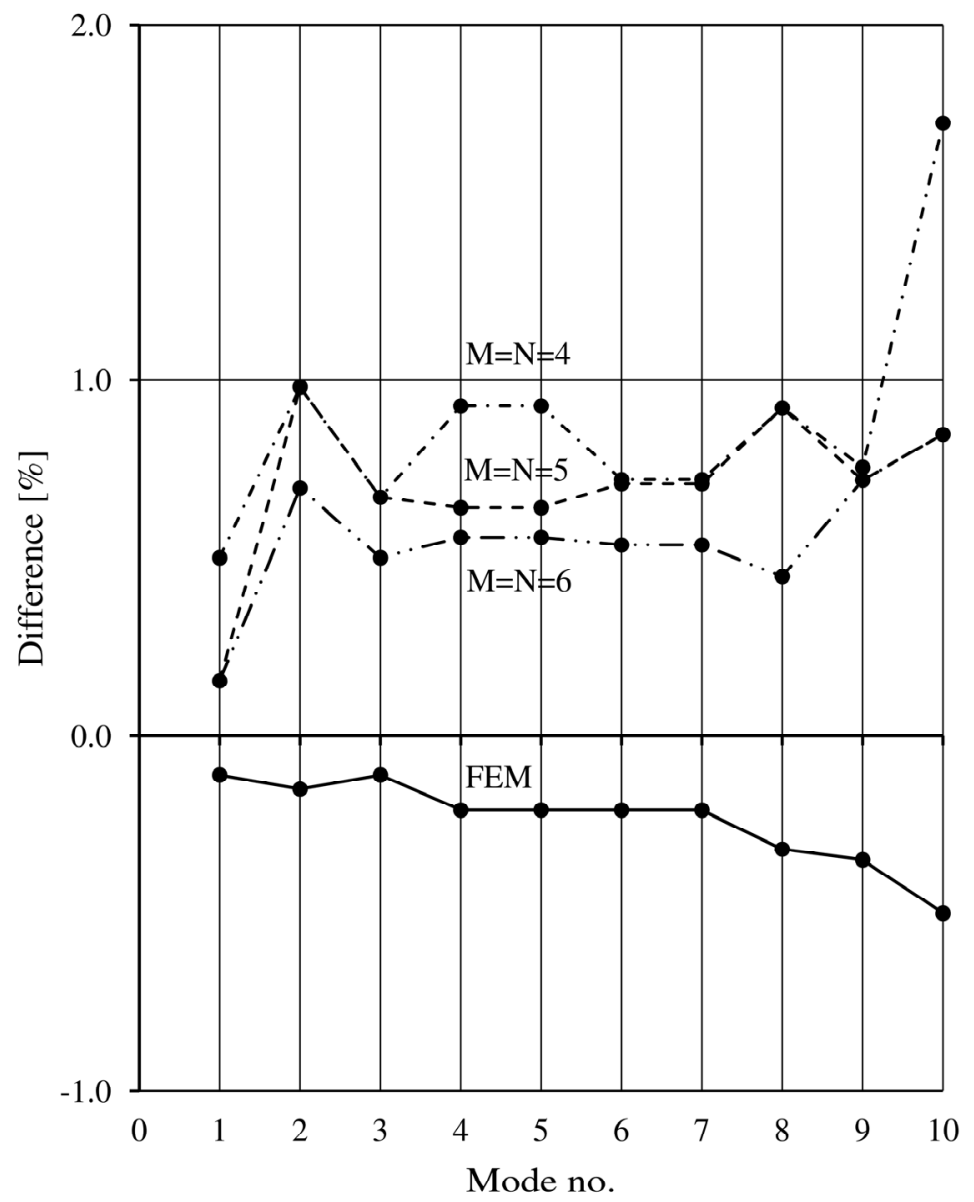

Fig. 6 Convergence of the DP-OMm procedure 
Table 3 Frequency parameter $\Omega=\omega(2 b / \pi)^{2} \sqrt{m / D}$ of a free square plate, DP-OMm, convergence analysis

\begin{tabular}{cccccccc}
\hline $\begin{array}{c}\text { Mode } \\
\text { No. }\end{array}$ & \multicolumn{2}{c}{$\mathrm{M}=\mathrm{N}=4$} & \multicolumn{2}{c}{$\mathrm{M}=\mathrm{N}=5$} & \multicolumn{2}{c}{$\mathrm{M}=\mathrm{N}=6$} & $\mathrm{RRM}$ \\
\hline 1 & $\Omega$ & $\%$ & $\Omega$ & $\%$ & $\Omega$ & $\%$ & Liew, [22] \\
\hline 2 & 2.3714 & 0.501 & 1.3667 & 0.153 & 1.3667 & 0.153 & 1.3646 \\
3 & 2.4755 & 0.671 & 2.4755 & 0.671 & 2.4713 & 0.501 & 2.4590 \\
4 & 3.5587 & 0.928 & 3.5486 & 0.642 & 3.5457 & 0.558 & 3.5260 \\
5 & 3.5587 & 0.928 & 3.5486 & 0.642 & 3.5457 & 0.558 & 3.5260 \\
6 & 6.2347 & 0.722 & 6.2339 & 0.709 & 6.2232 & 0.537 & 6.1900 \\
7 & 6.2347 & 0.722 & 6.2339 & 0.709 & 6.2232 & 0.537 & 6.1900 \\
8 & 6.5121 & 0.923 & 6.5121 & 0.923 & 6.4815 & 0.448 & 6.4526 \\
9 & 7.0710 & 0.756 & 7.0684 & 0.720 & 7.0684 & 0.720 & 7.0179 \\
10 & 7.9537 & 1.723 & 7.8853 & 0.849 & 7.8853 & 0.849 & 7.8190 \\
\hline
\end{tabular}

If frequency parameters are determined by the Rayleigh-Ritz method in the usual way by employing only ordinary mathematical modes, almost the same accuracy is achieved as in the case of DP-OMm provided that the same number of series terms is taken into account. However, the application of the one step iteration procedure results in a scarce spectrum of natural frequencies due to missing modes.

\section{Conclusion}

An analytical solution to rectangular plate natural vibrations can be achieved in a relatively simple way only in the case of at least two opposite simply supported edges. For all other combinations of boundary conditions, a numerical procedure, such as the Rayleigh-Ritz method or FEM, is used. In order to reduce the number of polynomial terms in the usual application of the Rayleigh-Ritz method, the beam mode functions $X_{m}$ and $Y_{n}$, satisfying the grillage boundary conditions, are often used as coordinate functions in the form of products, $W_{m n}=X_{m} Y_{n}$, which makes the separation of variables possible.

As shown in this paper, for a free rectangular plate, the modes $W_{m n}=X_{m} Y_{n}$ are only one family of possible modes shapes. Two additional mode families of the type $W_{m n \pm k l}=X_{m} Y_{n} \pm X_{k} Y_{l}$ are identified by analysing the complete natural response determined by FEM. These missing natural modes generate two very close frequency spectra, increasing in such a way the density of the resulting natural spectrum. If Poisson's ratio is zero and if there is no torsion, two frequency spectra are identical. This phenomenon of double frequency spectrum is also known in the natural vibrations of the simply supported Timoshenko beam, [23].

In the case of free square plate natural vibrations, the missing natural modes start from the very beginning of the frequency spectrum, while in that of a rectangular plate, they are shifted to higher frequencies by the increased value of the plate aspect ratio $a / b$. By recognizing the missing modes, the confusion of the square plate mode identification is 
cleared up. Natural frequencies determined by the Rayleigh quotient are approximate since the assumed modes are close but not equal to the real natural modes. However, their accuracy is acceptable for a preliminary design purpose.

Accuracy of the estimated natural frequencies can be increased by employing the Rayleigh-Ritz method and the approximate ordinary and missing modes as the mathematical ones in the governing series. In that way, the mass matrix is diagonal due to the orthogonality of the constitutive beam modes, while the stiffness matrix is full with dominant diagonal elements. As a result, only a slightly higher number of mathematical modes than the number of the desired accurate natural frequencies have to be taken into account. Moreover, the one step iteration procedure gives highly accurate results making in such a way the presented method superior to the known solutions.

The presented procedure can be used for the hydroelastic analysis of very large floating structures (VLFS), which behave as a sheet in the sea waves, [24]. An analytical formulation of the assumed modes simplifies the determination of hydrodynamic coefficients, i.e. added mass and hydrodynamic damping. This is illustrated in the case of a transient response of a very large floating airport with length and breadth of $L \times B=5 \times 1 \mathrm{~km}$, caused by the take-off and landing airplane loads, [25].

The analytical solution to free plate natural vibrations outlined in the paper can be readily applied in the qualitative analysis of complex fluid-structure interaction phenomena such as aeroelasticity (divergence and flutter of compliant flat plates), [26], and aeroacoustics (vibro-acoustic formulations of baffled flat plates), [27].

\section{Acknowledgment}

This study was supported by a National Research Foundation (NRF) of Korea grant awarded by the Korean Government (MSIP) through GCRC-SOP (Grant No. 2011-0030013).

\section{Nomenclature}

\begin{tabular}{ll|ll}
$a, b, h$ & $\begin{array}{l}\text { plate length, breadth, } \\
\text { thickness }\end{array}$ & $U$ & strain energy \\
$A_{i}$ & series coefficient & $W_{m n}$ & plate natural modes \\
$D$ & flexural rigidity & $X_{m}, Y_{n}$ & $\begin{array}{l}\text { beam natural modes } \\
\text { deflection function }\end{array}$ \\
$E$ & Young's modulus & $Z$ & $\begin{array}{l}\text { coefficient of trigonometric } \\
\text { and hyperbolic functions }\end{array}$ \\
$K$ & kinetic energy & $\alpha_{m}, \beta_{n}$ & Poisson's ratio \\
$m$ & mass per unit area & $v$ & natural frequency \\
$M_{i j}^{0}$ & $\begin{array}{l}\text { elements of modal mass } \\
\text { matrix }\end{array}$ & $\omega$ & \\
$S_{i j}^{0}$ & $\begin{array}{l}\text { elements of modal stiffness } \\
\text { matrix }\end{array}$ & &
\end{tabular}




\section{REFERENCES}

[1] G. Kirchhoff: Vorlesungen über Mathematische Physik, Vol. 1, B. G. Teubner, Leipzig, 1876.

[2] R. D. Mindlin: Influence of Rotary Inertia and Shear on Flexural Motions of Isotropic Elastic Plates, Journal of Applied Mechanics 18(1), 31-38, 1951.

[3] O. Hughes, J. K. Paik: Ship Structural Analysis and Design, SNAME, Jersey City, 2010.

[4] K. Inoue: Mega Float: Achievements of Date and Ongoing Plan of Research, The 9th ISOPE, Brest, France, Vol. 1, pp. 1-10, 1999.

[5] R. Szilard: Theories and Applications of Plate Analysis, John Wiley \& Sons, New York, 2004. DOI: 10.1002/9780470172872

[6] K. M. Liew, Y. Xiang, S. Kitipornchai: Research on Thick Plate Vibration: a Literature Survey, Journal of Sound and Vibration, 180, 163-176, 1995. DOI: 10.1006/jsvi.1995.0072

[7] I. Senjanović, N. Vladimir, M. Tomić: An advanced theory of moderately thick plate vibrations, Journal of Sound and Vibration, 332, 1868-1880, 2013. DOI: 10.1016/j.jsv.2012.11.022

[8] Y. Xing, B. Liu: Closed form solutions for free vibrations of rectangular Mindlin plates, Acta Mechanica Sinica, Vol. 25, no. 5, pp. 689-698, 2009. DOI: 10.1007/s10409-009-0253-7

[9] I. Senjanović, M. Tomić, N. Vladimir, D.S. Cho: Analytical solution for free vibrations of a moderately thick rectangular plate, Hindawi Publishing Corporation, Mathematical Problems in Engineering, Volume 2013, Article ID 207460, 13 pages, DOI: 10.1155/2013/207460.

[10] A. W. Leissa: The free vibration of rectangular plates, Journal of Sound and Vibration, 31, 257-293, 1973. DOI: $10.1016 / \mathrm{S} 0022-460 X(73) 80371-2$

[11] T. Mizusawa: Natural frequencies of rectangular plates with free edges, Journal of Sound and Vibration, 105, 451-459, 1986. DOI: 10.1016/0022-460X(86)90171-9

[12] M. Malik, C. W. Bert: Implementing multiple boundary conditions in the DQ solution of higher-order PDE's: application to free vibration of plates. International Journal for Numerical Methods in Engineering, 39, 1237-1258, 1996. DOI: 10.1002/(SICI)1097-0207(19960415)39:7<1237::AIDNME904>3.0.CO;2-2

[13] Y. Wang, X. Wang, Y. Zhou: Static and free vibration analyses of rectangular plates by the new version of differential quadrature element method, International Journal for Numerical Methods in Engineering, 59, 1207-1226, 2004. DOI: 10.1002/nme.913

[14] S. Zhao, G.W. Wei, Y. Xiang: DSC analysis of free-edged beams by an iteratively matched boundary method, Journal of Sound and Vibration, 284, 487-493, 2005. DOI: 10.1016/j.jsv.2004.08.037

[15] S.N. Yu, Y. Xiang, G.W. Wei: Matched interface and boundary (MIB) method for the free vibration analysis of plates, Communications in Numerical Methods in Engineering, 25, 923-950, 2009. DOI: $10.1002 / \mathrm{cnm} .1130$

[16] X. Wang, S. Xu: Free vibration analysis of beams and rectangular plates with free edges by the discrete singular convolution, Journal of Sound and Vibration, 329, 1780-1792, 2010. DOI: $10.1016 /$ j.jsv.2009.12.006

[17] C.M. Wang, Y. Xiang: Utsunomiya, T., Watanabe, E., Evaluation of modal stress resultants in freely vibrating plates, International Journal of Solids and Structures, 38, 6525-6558, 2001. DOI: $10.1016 / \mathrm{S} 0020-7683(01) 00040-3$

[18] MSC, 2005. MSC. NASTRAN 2005: Installation and Operations Guide. MSC Software.

[19] C.M. Wang, W.X. Wu, C. Shu, T. Utsunomiya: LSFD method for accurate vibration modes and modal stress-resultants of freely vibrating plates that model VLFS, Computers and Structures, 84, 2329-233, 2006. DOI: 10.1016/j.compstruc.2006.08.055

[20] I. Senjanović, M. Tomić, N. Vladimir, N. Hadžić: An approximate analytical procedure for natural vibration analysis of free rectangular plates, Thin-Walled Structures 95, 101-114, 2015. DOI: $10.1016 /$ j.tws.2015.06.015

[21] D.E. Newland: Mechanical Vibration Analysis and Computation, Dover Publications, Inc, Mineola, N.Y., 2006.

[22] K. M. Liew, Y. Xiang, S. Kitipornchai: Transverse Vibration of Thick Rectangular Plates - I. Comprehensive Set of Boundary Conditions, Computers \& Structures, Vol. 49, No. 1, pp. 1-29, 1993. DOI: $10.1016 / 0045-7949(93) 90122-\mathrm{T}$ 
[23] I. Senjanović, N. Vladimir: Physical Insight into Timoshenko Beam Theory and its Modification with Extension, Structural Engineering and Mechanics, Vol. 48, No. 4, 519-545, 2013.

DOI: 10.12989/sem.2013.48.4.519

[24] M. Kashiwagi: Transient response of a VLFS during loading and take-off of an airplane, Journal of Marine Science and Technology 9, 2004, pp. 14-23. DOI: 10.1007/s00773-003-0168-0

[25] I. Senjanović, M. Tomić, N. Vladimir: An advanced procedure for hydroelastic analysis of very large floating airport exposed to airplane load, Proceeding of the 7th International Conference on Hydroelasticity in Marine Technology, Split, Croatia, 2015.

[26] A.D. Lucey, P.W. Carpenter: The hydroelastic stability of three-dimensional disturbances of a finite compliant wall, Journal of Sound and Vibration 165(3) (1993), 527-552. DOI: 10.1006/jsvi.1993.1275

[27] P. Vitiello, S. De Rosa, F. Franco: Convected field analysis of flat panels response to turbulent boundary layer induced vibration, Aerospace Science and Technology 12 (2008), 91-104.

DOI: $10.1016 /$ j.ast.2007.10.003

Submitted: $\quad 02.02 .2016$

Accepted: $\quad 20.9 .2016$
Ivo Senjanović

Marko Tomić

Nikola Vladimir

Neven Hadžić

University of Zagreb,

Faculty of Mechanical Engineering and

Naval Architecture,

Ivana Lučića 5,

10000 Zagreb, Croatia

e-mail: ivo.senjanovic@fsb.hr 